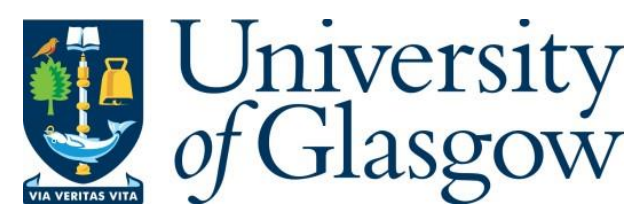

Tamvakis, A., Anagnostopoulos, C.-N., Tsirtsis, G., Niros, A. D. and Spatharis, S. (2018) Optimized classification predictions with a new index combining machine learning algorithms. International Journal on Artificial Intelligence Tools, 27(3), 1850012.

There may be differences between this version and the published version. You are advised to consult the publisher's version if you wish to cite from it.

http://eprints.gla.ac.uk/163013/

Deposited on: 13 September 2018

Enlighten - Research publications by members of the University of Glasgow http://eprints.gla.ac.uk 


\title{
Optimized classification predictions with a new index combining machine learning algorithms
}

\author{
Androniki Tamvakis \\ Department of Marine Sciences, University of the Aegean, University Hill, \\ Mytilene, 81100, Greece \\ atamvaki@mar.aegean.gr
}

Christos-Nikolaos Anagnostopoulos

\author{
Department of Cultural Technology and Communication, University of the Aegean, University Hill, \\ Mytilene, 81100, Greece
}

canag@ct.aegean.gr

George Tsirtsis

Department of Marine Sciences, University of the Aegean, University Hill,

Mytilene, 81100, Greece

gtsir@aegean.gr

Antonios D. Niros

Department of Cultural Technology and Communication, University of the Aegean, University Hill, Mytilene, 81100, Greece

aneiros@aegean.gr

Sofie Spatharis

Institute of Biodiversity, Animal Health and Comparative Medicine, University of Glasgow, Glasgow, Scotland, G12 8QQ, UK

Sofie.Spatharis@glasgow.ac.uk

Voting is a commonly used ensemble method aiming to optimize classification predictions by combining results from individual base classifiers. However, the selection of appropriate classifiers to participate in voting algorithm is currently an open issue. In this study we developed a novel Dissimilarity-Performance (DP) index which incorporates two important criteria for the selection of base classifiers to participate in voting: their differential response in classification (dissimilarity) when combined in triads and their individual performance. To develop this empirical index we firstly used a range of different datasets to evaluate the relationship between voting results and measures of dissimilarity among classifiers of different types (rules, trees, lazy classifiers, functions and Bayes). Secondly, we computed the combined effect on voting performance of classifiers with different individual performance and/or diverse results in the voting performance. Our DP index was able to rank the classifier combinations according to their voting performance and thus to suggest the optimal combination. The proposed index is recommended for individual machine learning users as a preliminary tool to identify which classifiers to combine in order to achieve more accurate classification predictions avoiding computer intensive and time-consuming search.

Keywords: voting ensemble method; classification; classifier dissimilarity; individual performance; classifier ensemble index. 


\section{Introduction}

Machine Learning (ML) refers to the construction and study of algorithms that acquire information from collected data aiming to the most effective generalization and representation of the scientific issue under consideration. ${ }^{1}$ Classification is one of the most common ML applications, whereby an output variable (target class) with discrete and unordered values (labels) is predicted from a set of collected samples (instances) that consist the training set (dataset). ${ }^{2}$ This approach spans cutting edge applications over a wide variety of scientific fields ${ }^{3,4}$ such as bioinformatics, ${ }^{5}$ medical science, ${ }^{6}$ computing, ${ }^{7}$ astronomy, ${ }^{8}$ engineering, ${ }^{9}$ remote sensing ${ }^{10,11}$ and environmental science. ${ }^{12}$

The increased interest in classification resulted in the development of numerous classifiers, differentiated in supervised or unsupervised depending on whether the training dataset is labeled a priori or not. ${ }^{13}$ Among the most popular supervised classifiers are decision trees, multilayer perceptron, naïve Bayes classifiers, instance based learning and support vector machines. ${ }^{14}$ Despite their wide variety, no optimal classifier has been established so far. ${ }^{15}$ Instead, the classification performance depends on the dataset properties (e.g. input variables, number of training samples) $)^{11,16}$ or the method used to assess classifier performance. ${ }^{17}$

Current research on ML focuses on integrating optimal classification results from multiple classifiers using specialized techniques called ensemble methods (EMs). ${ }^{18-20}$ These methods provide significantly improved classification performance compared to the base classifiers. . $^{18,19,21,22}$ Voting is a particularly useful and comprehensive EM that collects votes (i.e. predicted labels of the target class) from multiple classifiers and predicts the label of the target class yielding the highest value (expressed as number of votes or probability). Voting is the most widely applicable EM method, while other EMs (including bagging and boosting) are also based on voting to provide classification. ${ }^{23,24}$ Voting is the simplest and easiest way to combine classifiers, ${ }^{25-27}$ demanding no extra training for final prediction except for the pre-existing individual classifier classifications. ${ }^{28}$ Due to its ability to significantly improve predictions, voting spans many applications ranging from simple classification tasks ${ }^{29,30}$ to more complex implementations such as clustering, ${ }^{31}$ pairwise comparison ${ }^{32}$ and fuzzy systems. ${ }^{33,34}$

The challenging step when employing a voting algorithm is the selection of the base classifiers to be combined. When the number of potential classifier combinations and the size of the dataset are rather small, the optimal classifier combination can be determined exhaustively. However, this search becomes increasingly labor intensive and time consuming as the dataset and classifier complexity increase. ${ }^{35}$ To simplify this process, appropriate criteria must be applied for the selection of optimal classifiers to participate in the voting algorithm. For instance when classifiers in a voting scheme are highly dissimilar or independent (as assessed with dissimilarity indices), the classification performance may be significantly improved since the misclassification of one classifier can be potentially corrected by the success of another. ${ }^{36-39}$ Previous studies tried to incorporate the classifiers' diversity in order to construct successful ensembles by using 
different methods such as clustering, pruning, proper weighting based on various diversity measures, fuzzy logic, greedy search, particle swarm optimization, random sampling or data manipulation. ${ }^{19,20,23,26,40-43}$

Another important criterion is the performance of individual base classifiers to be combined during voting. Although it would seem intuitive to combine the best performing classifiers during voting, previous studies have shown that the best classification is not always achieved by combining classifiers that show the best individual performance. ${ }^{35,44,45}$ Therefore, the selection of the best combination of base classifiers should be based on simple and flexible criteria that will jointly consider their dissimilarity or independence along with their individual classification performance. ${ }^{26,46-}$ 48

In this study we aim to develop a user-friendly index capable of identifying the optimal combination of base classifiers feeding into the voting algorithm and maximizing its classification performance. To this aim, the specific objectives are (a) to assess the efficiency of base classifiers in performing substantially different classification tasks, (b) to identify combinations of base classifiers that have markedly different behavior (i.e. high dissimilarity), (c) to test whether these combinations also have a corresponding high performance in voting classification, and (d) to develop a new user-friendly index that will incorporate the two criteria of classifier dissimilarity and individual performance, by assigning them appropriate weights. We expect that this dissimilarity-performance (DP) index will be more efficient in selecting the most appropriate base classifiers to be combined for voting than the traditionally applied dissimilarity indices. In order to subjectively evaluate its efficiency, DP will be confronted with substantially different classification tasks based on 28 (training and testing) datasets spanning different scientific fields.

\section{Methods}

\subsection{Outline of the Methodology}

The development of the DP index, identifying the optimal combination of base classifiers to perform classification tasks, was carried out as follows (Fig. 1). Initially 10 base classifiers were trained in order to directly compare their individual performance in 14 different classification tasks (training datasets). This information was later used in the development of the new index which incorporates the individual performance of the base classifiers. The next step involved the training of the voting algorithm with all possible combinations of the 10 base classifiers in triads using all datasets. Thus, for each dataset the voting EM was trained using 120 different triads of base classifiers (denoted by all combinations of 3 out of the 10 selected base classifiers) and the voting performance of each triad was specified. Then, Binary Dissimilarity Indices (BDIs) were computed for all possible classifier triads of the training datasets to quantify within triad dissimilarity, and this measure was correlated with the corresponding voting performance. The last step 
was the development of the new DP index that takes into account both the individual performance of each base classifier and the dissimilarity of classifiers within triads used in voting. These two characteristics were optimally weighted using a weighing parameter $a$ for all training datasets. To check whether DP efficiently rates the classifier combinations according to their voting performance, the DP classification performance was correlated with the corresponding voting performance of triads across the 14 testing datasets. To test whether DP can identify the optimal classifier triad we checked whether the optimal triad based on voting performance was amongst those that DP identified as the best classifier combinations. Finally, the overall behavior of DP was examined across all testing datasets, to assess its efficiency and consistency to perform substantially different classification tasks.

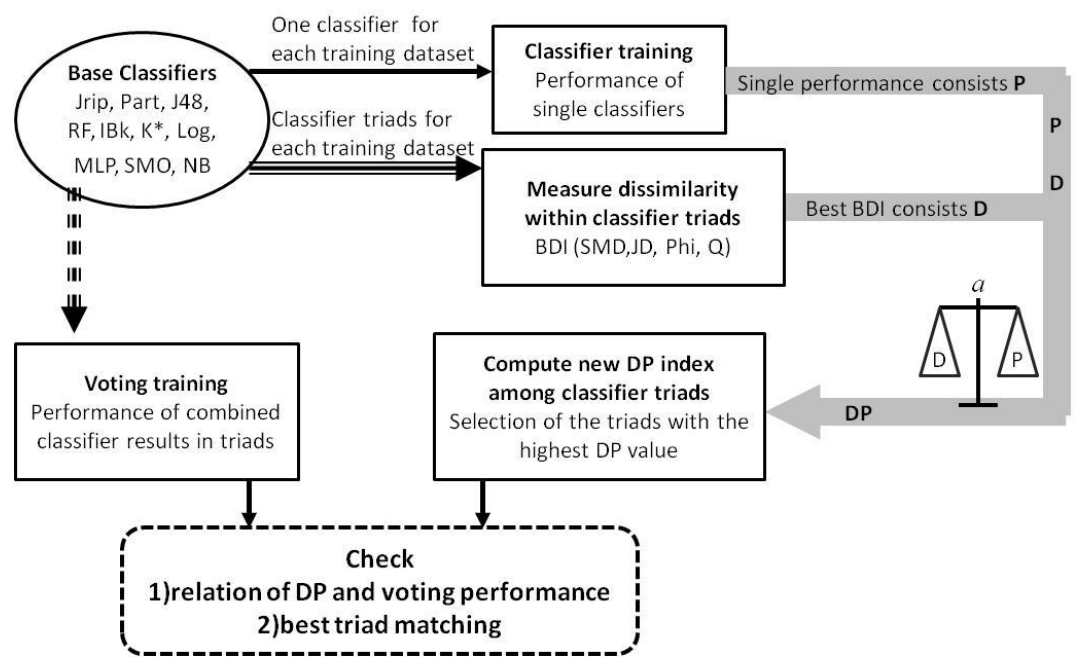

Fig. 1. Schematic diagram of the methodological steps followed in the development and testing of the proposed Dissimilarity-Performance (DP) index. This index takes into account both the individual performance of base classifiers (P) and the dissimilarity (D) of classifier performance -measured with Binary Dissimilarity Indices (BDIs) - when those are combined in triads.

All base classifiers were trained with the WEKA machine learning package (version 3.6.9). ${ }^{49}$ This package was also used for training the voting algorithm with all possible combinations of classifiers in triads. The purpose of DP index is to identify the optimal combination of classifiers maximizing the classification performance during voting and therefore it is not necessary for base classifiers to participate with their highest potential performance. For this reason, the training of each classifier was performed using the default parameter values of the WEKA package.

\subsection{Datasets}


Twenty eight datasets (Table 1) were randomly split into two groups. The first group was used for the training of base classifiers and voting EM in order to determine the weight of the DP index parameter $a$ (Fig. 1). Twelve datasets of the first group are available from the UCI machine learning repository and two are related with the research interests of the authors. The first of those is the Berlin Emotional (BER) database (EMO-DB) ${ }^{50}$ which contains 535 utterances of 10 actors ( 5 male, 5 female) predicting 7 emotional states (anger, happiness, anxiety/fear, sadness, boredom, disgust and neutral). After processing with PRAAT software ${ }^{51}$ each utterance was converted to a 133-dimensional prosodic feature vector based on well-established speech features, such as Pitch, Mel Frequency Cepstral Coefficients (MFCCs), energy and formant frequencies. ${ }^{52}$ Thus, the dataset consists of 535 samples with 133 prosodic input variables to be categorized in 7 class labels. The second dataset (ECO) comprises of 188 seawater samples collected on monthly campaigns during one annual cycle (August '04-July '05) in Kalloni Gulf, Lesvos Island, Greece. ${ }^{53}$ The dataset includes 9 physico-chemical input variables (e.g. temperature and nutrients) and one target class including 5 levels of ecological quality (high, good, moderate, poor and bad). Those levels are based on chlorophyll $\alpha$ limits set by Simboura et al. ${ }^{54}$ for the evaluation of coastal water ecological quality for the purposes of the European Water Framework Directive 2000/60/EC.

Table 1. List of datasets used in the experiments along with their structural characteristics i.e. number of instances and input variables, type of input variables (c for categorical, i for integer, $r$ for real), number of labels of the target class and indicative classification performance based on previous studies.

\begin{tabular}{|c|c|c|c|c|c|c|}
\hline & & Dataset Name & $\begin{array}{c}\text { No. of } \\
\text { instances }\end{array}$ & $\begin{array}{c}\text { No. and type } \\
\text { of input } \\
\text { variables }\end{array}$ & $\begin{array}{c}\text { No. of labels } \\
\text { of the target } \\
\text { class }\end{array}$ & $\begin{array}{c}\text { Previous } \\
\text { classification } \\
\text { performance }\end{array}$ \\
\hline \multicolumn{7}{|c|}{ Training Datasets } \\
\hline \multirow{14}{*}{ 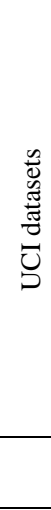 } & 1. & Arrhythmia (ARR) & 452 & $279(\mathrm{c}, \mathrm{i}, \mathrm{r})$ & 16 & $71.25^{55}$ \\
\hline & 2. & Breast cancer (BRE) & 286 & $9(\mathrm{c})$ & 2 & $76.63^{55}$ \\
\hline & 3. & German credit (GER) & 1,000 & $20(\mathrm{c}, \mathrm{i})$ & 2 & $75.50^{56}$ \\
\hline & 4. & Glass Identification (GLA) & 214 & $9(\mathrm{r})$ & 6 & $65.50^{57}$ \\
\hline & 5. & Ionosphere (ION) & 351 & $34(\mathrm{i}, \mathrm{r})$ & 2 & $93.00^{58}$ \\
\hline & 6. & Iris (IRI) & 150 & $4(\mathrm{r})$ & 3 & $97.00^{59}$ \\
\hline & 7. & Labor relations (LAB) & 57 & $16(\mathrm{c}, \mathrm{i}, \mathrm{r})$ & 2 & $89.27^{60}$ \\
\hline & 8. & Page blocks (PAG) & 5,473 & $10(\mathrm{i}, \mathrm{r})$ & 5 & $91.80^{59}$ \\
\hline & 9. & Segment challenge (SEG) & 1,500 & $19(\mathrm{r})$ & 7 & $96.93^{61}$ \\
\hline & 10. & Vehicle silhouettes (VEH) & 946 & 18 (i) & 4 & $75.00^{62}$ \\
\hline & 11. & Vote (VOT) & 435 & $16(\mathrm{c})$ & 2 & $97.00^{63}$ \\
\hline & 12. & Waveform (WAV) & 5,000 & $40(\mathrm{r})$ & 3 & $83.16^{64}$ \\
\hline & 13. & Berlin emotional database (BER) & 535 & $133(\mathrm{r})$ & 7 & $78.00^{51}$ \\
\hline & 14. & Ecological quality of Kalloni Gulf (ECO) & 188 & $9(\mathrm{r})$ & 5 & - \\
\hline \multicolumn{7}{|c|}{ Testing Datasets } \\
\hline \multirow{6}{*}{ 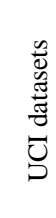 } & 1. & Banknote authentication (BAN) & 1,372 & $4(r)$ & 2 & $100.00^{65}$ \\
\hline & 2. & Blogger (BLO) & 100 & $5(\mathrm{c})$ & 3 & $85.00^{66}$ \\
\hline & 3. & Car evaluation (CAR) & 1,728 & $5(\mathrm{c})$ & 3 & $95.78^{56}$ \\
\hline & 4. & Chronic kidney disease (CHR) & 400 & $24(\mathrm{c}, \mathrm{r})$ & 2 & $99.30^{67}$ \\
\hline & 5. & Dermatology (DER) & 366 & $34(\mathrm{c}, \mathrm{r})$ & 6 & $98.40^{59}$ \\
\hline & 6. & Fertility (FER) & 100 & $9(\mathrm{c}, \mathrm{r})$ & 2 & $86.00^{68}$ \\
\hline
\end{tabular}




\begin{tabular}{rlcccc}
\hline 7. & Forest type mapping (FOR) & 324 & $27(\mathrm{r})$ & 4 & $85.90^{69}$ \\
8. & Hepatitis (HEP) & 155 & $19(\mathrm{c}, \mathrm{i}, \mathrm{r})$ & 2 & $85.80^{55}$ \\
9. & Lung cancer (LUN) & 32 & $56(\mathrm{i})$ & 3 & $63.84^{70}$ \\
10. & Seeds (SEE) & 210 & $7(\mathrm{r})$ & 3 & $92.00^{71}$ \\
11. & Seismic bumps (SEI) & 2,584 & $18(\mathrm{c}, \mathrm{r})$ & 2 & $93.30^{72}$ \\
12. & Teaching assistant evaluation (TEA) & 151 & $5(\mathrm{c}, \mathrm{i})$ & 3 & $52.50^{59}$ \\
13. & Wine quality (WIN) & 4,898 & $11(\mathrm{r})$ & 7 & $64.60^{73}$ \\
\hline 14. & Abdominal pain (ABD) & 516 & $16(\mathrm{c}, \mathrm{i}, \mathrm{r})$ & 7 & $86.04^{55}$ \\
\hline
\end{tabular}

The second group of datasets was used to evaluate the performance of the newly proposed DP index into unseen data. Thirteen of those datasets originate from the UCI machine learning repository while the last one (i.e. Abdominal pain) is related to the authors' research. ${ }^{74}$ Abdominal pain (ABD) dataset contains 512 children's medical records consisting of 16 demographic, clinical and laboratory input variables (e.g. sex, age, duration of pain, temperature, existence of anorexia or neutrophilia). The target class contains seven possible diagnosis results (i.e. focal appendicitis, phlegmonous or supurative appendicitis, gangrenous appendicitis, peritonitis, observation, discharge and no findings).

The datasets of both groups (training and testing) originating from various scientific fields (e.g. Medicine, Ecology, Botany, Physics, Sociology, Economy, Web, and Engineering), deal with different classification problems leading to varied accuracies (classification performance from $65.50 \%$ to $97.00 \%$ for the training and $52.50 \%$ to $100.00 \%$ for the testing group). This is due to the sufficiently different structure and interactions of database variables. Indeed, many characteristics substantially differ amongst those datasets, such as the number of instances (57 to 5,473 for the training and 32 to 4,898 for the testing group), type (categorical, integer or real) and number of input variables (4 to 279 for the training and 4 to 56 for the testing group), predicted labels of the target class ( 2 to 16 for the training and 2 to 7 for the testing group). Additionally, all datasets differ in structure and functionality as some of them have relatively low numbers of instances compared to the number of input variables (LAB, BER or LUN), others contain numerous instances (PAG, WAV or WIN) whereas others have many class labels compared to the available training instances (ARR, DER or FOR) or vice versa (SEG or BAN). This diversity in database characteristics is expected to result in substantial differences in the performance of classifiers and their quantified dissimilarity supporting the exhaustive training and testing of the existing indices and the currently proposed DP index.

\subsection{Training of base classifiers and voting $E M$}

The 10 base classifiers were selected to represent all different categories of classification such as rules, trees, lazy classifiers, functions, and Bayes (Table 2). The voting EM combines the results of base classifiers ${ }^{15,40}$ in triads to provide a classification for all instances of the 28 databases. In this work, an exhaustive training of the voting algorithm was achieved by combining the 10 base classifiers in all possible triads (i.e. 120 different combinations). We used classifier triads because the combination of an odd number of classifiers during voting excludes the risk of ties. ${ }^{36}$ Additionally, three is the minimum 
odd number that can be used in voting and thus combining classifiers in triads simplifies the whole procedure with respect to complexity and processing time. Three base classifier combinations have been commonly used in various EMs applications. . $^{37,39,47,75-77}$

Table 2. Base algorithms plus voting ensemble method with their default WEKA values used during their training

\begin{tabular}{|c|c|c|c|}
\hline Category & Abbreviation & Classifier description & Default WEKA values \\
\hline \multirow[t]{2}{*}{ Rules } & $\mathrm{JRip}^{78}$ & $\begin{array}{l}\text { Implements the repeated } \\
\text { incremental pruning to produce } \\
\text { error reduction (RIPPER) }\end{array}$ & $\begin{array}{l}\text { Minimum weight of the } \\
\text { instances in a rule }=2\end{array}$ \\
\hline & Part $^{79}$ & Generates a PART decision list & $\begin{array}{l}\text { Minimum N\# of instances } \\
\text { per rule }=2\end{array}$ \\
\hline \multirow[t]{2}{*}{ Trees } & $\mathrm{J} 48^{80}$ & Generates a $\mathrm{C} 4$ decision tree & $\begin{array}{l}\text { Minimum N\# of instances } \\
\text { per leaf }=2\end{array}$ \\
\hline & $\mathrm{RF}^{81}$ & $\begin{array}{l}\text { Constructs a forest of random } \\
\text { trees }\end{array}$ & $\begin{array}{l}\text { Maximum depth }=\text { unlimited, } \\
\text { N\# of trees }=10\end{array}$ \\
\hline \multirow[t]{2}{*}{ Lazy } & $\mathrm{IBk}^{82}$ & The $k$ nearest neighbor & $k=1$ \\
\hline & $\mathrm{K}^{* 83}$ & $\begin{array}{l}\text { Instance-based with entropic } \\
\text { distance measure }\end{array}$ & $\begin{array}{l}\text { Parameter of global blending } \\
=20\end{array}$ \\
\hline \multirow[t]{3}{*}{ Functions } & $\log ^{84}$ & Multinomial logistic regression & - \\
\hline & $\mathrm{MLP}^{85}$ & $\begin{array}{l}\text { Multilayer Perceptron trained with } \\
\text { backpropagation }\end{array}$ & 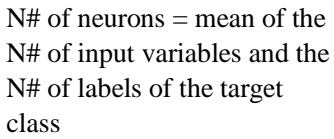 \\
\hline & $\mathrm{SMO}^{86}$ & $\begin{array}{l}\text { Sequential Minimal Optimization } \\
\text { for training a support vector } \\
\text { classifier }\end{array}$ & Complexity parameter = 1 \\
\hline Bayes & $\mathrm{NB}^{87}$ & $\begin{array}{l}\text { The Naïve Bayes classifier using } \\
\text { estimator classes }\end{array}$ & - \\
\hline Meta & Vote $^{88}$ & $\begin{array}{l}\text { Algorithm for combining } \\
\text { classifier results }\end{array}$ & $\begin{array}{l}\text { Combination rule }=\text { average } \\
\text { of probabilities }\end{array}$ \\
\hline
\end{tabular}

The efficiency of the 10 base classifiers and the voting algorithm was evaluated for all datasets using the 10 -fold cross validation procedure. ${ }^{89}$ The voting EM was trained based on the averaged probability estimates of the base classifiers, resulting to the classical weighted voting schema which for each instance gives to the combining classifiers the power of their individual performance related to the class they propose. ${ }^{20,90}$ Weighted training usually outmatches majority voting (i.e. the predicting class label needs to take at least half votes) or plurality voting (i.e. the predicting class label takes the largest number of votes) while it optimally confronts the confusing situation of ties. Thus, when the three classifiers vote a different class label, the weighted voting predicts the label of the more successful base classifier. For instance if the $1^{\text {st }}$ classifier votes for "A" label, the $2^{\text {nd }}$ votes for "B" and the $3^{\text {rd }}$ votes for "C", the voting EM searches for the rates of successfulness of each base classifier for the specific predicting labels. Then if the $1^{\text {st }}$ classifier predicts the " $\mathrm{A}$ " label with $p_{1 A}=0.6$ rate of successfulness and the $2^{\text {st }}$ and $3^{\text {rd }}$ classifier predict the "B" and "C" label with $p_{2 B}=0.7$ and $p_{3 C}=0.8$ rates correspondingly, the voting EM will predict label " $\mathrm{C}$ " for the specific instance. In case 
the two best rates are equal, the label of the most overall successful classifier will be selected. ${ }^{20}$ The classification performance was assessed with the most commonly used criterion i.e. the percentage of Correctly Classified Instances (CCI).${ }^{14} \mathrm{CCI}$ is calculated as the percentage of the true positive and true negative predictions. Values of CCI higher than $70 \%$ are considered as reliable, however the efficiency of the classification performance depends greatly on the characteristics of each dataset. ${ }^{91}$

\subsection{Binary diversity indices (BDIs)}

BDIs quantify the dissimilarity or independency of classifications of base classifiers when they are combined in triads. This is later used to determine whether combinations of dissimilar or independent classifiers also have a corresponding high performance during voting. In the present study, the correct classification of an instance by a classifier was assigned a "1" score, whereas misclassification was assigned a " 0 " score. Using this binary assessment for all 10 classifiers, four well-known BDIs (Table 3) were computed. The first three BDIs are estimated by combining the classification results of two classifiers. Thus, to express dissimilarity with simple matching distance (SMD), Jaccard distance (JD) and independency (Phi) in triads, an average of the paired combinations was calculated. The last index $(\mathrm{Q})$, being also a measure of independency (positive or negative) between classifiers, is estimated by using three classifiers as described in Kuncheva et al..$^{76}$.

Table 3. Definition and ranges of four binary similarity (or dissimilarity) indices

\begin{tabular}{|c|c|c|c|}
\hline Coefficient & $\begin{array}{l}\text { Required } \\
\text { classifiers }\end{array}$ & Range & Formula $\left(S_{i j}\right.$ or $\left.S_{i j k}\right)$ \\
\hline \multirow{2}{*}{$\begin{array}{l}\text { Simple matching } \\
\text { distance (SMD) }\end{array}$} & \multirow{2}{*}{2} & \multirow{2}{*}[0,1]{} & $N^{10}+N^{01}$ \\
\hline & & & $\overline{N^{11}+N^{10}+N^{01}+N^{00}}$ \\
\hline \multirow{2}{*}{$\begin{array}{l}\text { Jaccard Distance } \\
(\mathrm{JD})^{93}\end{array}$} & \multirow{2}{*}{2} & \multirow{2}{*}[0,1]{} & $N^{10}+N^{01}$ \\
\hline & & & $\overline{N^{11}+N^{10}+N^{01}}$ \\
\hline \multirow{2}{*}{$\mathrm{Phi}^{94}$} & \multirow{2}{*}{2} & \multirow{2}{*}[-1,1]{} & $N^{11} N^{00}-N^{10} N^{01}$ \\
\hline & & & $\sqrt{\left(N^{11}+N^{10}\right)\left(N^{01}+N^{00}\right)\left(N^{10}+N^{00}\right)\left(N^{11}+N^{01}\right)}$ \\
\hline \multirow{2}{*}{$Q^{95}$} & \multirow{2}{*}{3} & \multirow{2}{*}[-1,1]{} & $N^{111} N^{001} N^{010} N^{100}-N^{011} N^{101} N^{110} N^{000}$ \\
\hline & & & $N^{111} N^{001} N^{010} N^{100}+N^{011} N^{101} N^{110} N^{000}$ \\
\hline \multicolumn{2}{|l|}{$N^{11}$} & \multicolumn{2}{|c|}{ Number of instances that have been correctly classified by both classifiers } \\
\hline \multicolumn{2}{|l|}{$N^{10}, N^{01}$} & \multicolumn{2}{|c|}{$\begin{array}{l}\text { Number of instances that have been correctly classified by the } 1^{\text {st }} \text { classifier but } \\
\text { not by the } 2^{\text {nd }} \text { and vice versa }\end{array}$} \\
\hline \multicolumn{2}{|l|}{$N^{00}$} & \multicolumn{2}{|c|}{ Number of instances that have been correctly classified by neither classifier } \\
\hline \multicolumn{2}{|l|}{$N^{111}$} & \multicolumn{2}{|c|}{ Number of instances that have been correctly classified by all three classifiers } \\
\hline \multicolumn{2}{|l|}{$N^{011}, N^{101}, N^{110}$} & \multicolumn{2}{|c|}{$\begin{array}{l}\text { Number of instances that have been correctly classified by the } 2^{\text {nd }} \text { and } 3^{\text {rd }} \\
\text { classifiers but not by } 1^{\text {st }} \text { and the other calculated in the same way }\end{array}$} \\
\hline \multicolumn{2}{|l|}{$N^{001}, N^{010}, N^{100}$} & \multicolumn{2}{|c|}{$\begin{array}{l}\text { Number of instances that have been correctly classified by } 3^{\text {rd }} \text { classifiers but } \\
\text { not by } 1^{\text {st }} \text { nor } 2^{\text {nd }} \text { and the other calculated in the same way }\end{array}$} \\
\hline \multicolumn{2}{|l|}{$N^{000}$} & \multicolumn{2}{|c|}{ Number of instances that have been correctly classified by neither classifier } \\
\hline
\end{tabular}




\subsection{Dissimilarity-Performance index (DP)}

Apart from the classifier dissimilarity within triads (quantified by the traditionally used BDIs), the individual performance of each classifier was also considered in the present study aiming to provide further information on the best set of classifiers to be combined for improved classification. ${ }^{96}$ This joint approach for the identification of the best performing classifier triad was achieved in the present study by developing an integrated Dissimilarity-Performance (DP) index. The proposed formula for this DP index is the following:

$$
D P=(1-a) \cdot \frac{\sum_{i<j}^{n} J_{i, j}}{3}+a \cdot \frac{\sum_{i=1}^{n} p_{i}}{3} \quad i, j=1,2,3
$$

where $J_{i, j}$ the JD index calculated from the binary classification results of the $i$-th and $j$-th classifiers, $p_{i}$ is the ratio of the correctly classified samples by the $i$-th classifier to the total number of instances and $a$ is a parameter between 0 and $1(0 \leq a \leq 1)$. The first addend represents the average of the JD for all classifier pairs, while the second is the average of the single performances of each classifier used in voting. Parameter $a$ expresses the different contribution of the aforementioned criteria, i.e. dissimilarity and performance of the combined classifiers. Therefore, if $a$ is equal to 0 the characteristic of dissimilarity among classifiers prevails in the DP index, expressing the case that better voting performance is achieved by combining classifiers showing large discrepancies among their individual predictions. On the other hand, if $a$ is equal to 1 only the individual performance of the base classifiers is counting in the DP index, meaning that the voting performance of the various classifier combinations is proportional solely to their individual performance. Finally, if $a=0.5$ then the two criteria have an equal contribution to the DP index. It should be noted that the DP values are always in the range 0 to 1 .

The efficiency of BDIs and DP based on the performance criterion (i.e. CCI) for the 120 different classifier triads was assessed with Spearman's rank correlation coefficient. Moreover, the DP index was calculated for many different values of the parameter $a$ (from 0 to 1 with a $0.01 \mathrm{step}$ ) for each classifier triad across the 14 training databases in order to define the value of $a$ that optimizes the Spearman's rank correlation coefficient between DP and voting performance. This procedure (a) optimizes the determining force of DP in identifying the best classifier triads, (b) determines the efficiency and consistency of DP to perform substantially different classification tasks (c) offers a prioritization of the two crucial criteria of dissimilarity and performance among classifiers participating in voting. Furthermore, we tested whether DP can identify the classifier triad with the best voting performance for 14 testing (unseen) datasets. This was performed by crosschecking whether the best triad falls within the $10 \%$ of the triads (i.e. 12 triads) with the highest DP values. DP was further tested for monotonicity (consistent increase or decrease along the CCI spectrum) as this is an important prerequisite for an index. ${ }^{97}$ Finally, the DP efficiency was examined to improve the voting EM when trained with datasets containing different number of labels of the target class. 


\section{Results}

\subsection{Results of base classifiers and voting ensemble method}

The classification performance of the worst and best performing base classifier along with the corresponding performance of the voting EM across all datasets are presented in Table 4. The MLPs achieved the highest performance in most databases and can be characterized as the best base classifier in this study. On the other hand, NB was the base classifier with the lowest performance showing the lowest \% CCI in twelve out of 28 databases.

Table 4. Percentage of CCI for the worst and best base classifiers cited in parenthesis along with the corresponding percentage of the Voting EM for training databases. The symbol $\uparrow$ denotes the participation of the best base classifier in the best classifier triad of Voting EM.

\begin{tabular}{|c|c|c|c|c|}
\hline & Database & $\begin{array}{l}\text { Worst base classifier } \\
\text { performance }\end{array}$ & $\begin{array}{l}\text { Best base classifier } \\
\text { performance }\end{array}$ & $\begin{array}{l}\text { Voting performance } \\
\text { (best triad) }\end{array}$ \\
\hline \multirow{14}{*}{ 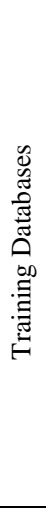 } & ARR & $52.88(\mathrm{IBk})$ & 70.35 (JRip) & $71.48(\dagger)$ \\
\hline & BRE & 64.69 (MLP) & $75.52(\mathrm{~J} 48)$ & $77.30(\dagger)$ \\
\hline & GER & $69.40\left(\mathrm{~K}^{*}\right)$ & $75.40(\mathrm{NB})$ & $76.90(\dagger)$ \\
\hline & GLA & $48.60(\mathrm{NB})$ & $75.23(\mathrm{RF})$ & $81.71(\dagger)$ \\
\hline & ION & $82.62(\mathrm{NB})$ & 92.88 (RF) & 94.89 \\
\hline & IRI & 94.00 (Part) & 97.33 (MLP) & $97.33(\dagger)$ \\
\hline & LAB & 82.46 (IBk) & $92.98(\mathrm{Log})$ & $96.67(\dagger)$ \\
\hline & PAG & $90.85(\mathrm{NB})$ & 97.01 (Part) & $97.50(\dagger)$ \\
\hline & SEG & $81.07(\mathrm{NB})$ & 96.80 (MLP) & $98.13(\dagger)$ \\
\hline & VEH & $44.80(\mathrm{NB})$ & 81.68 (MLP) & $83.23(\dagger)$ \\
\hline & VOT & $90.11(\mathrm{NB})$ & 96.32 (J48) & $97.01(\dagger)$ \\
\hline & WAV & $73.48\left(\mathrm{~K}^{*}\right)$ & 86.68(SMO) & $86.74(\dagger)$ \\
\hline & BER & $51.59(\mathrm{NB})$ & 81.70 (MLP) & $86.73(\dagger)$ \\
\hline & $\mathrm{ECO}$ & 44.15 (SMO) & $63.30(\mathrm{IBk})$ & 69.15 \\
\hline \multirow{14}{*}{ 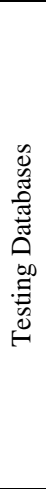 } & BAN & $84.26(\mathrm{NB})$ & 99.93 (MLP) & $100.00(\dagger)$ \\
\hline & BLO & $71.00(\mathrm{NB})$ & $85.00(\mathrm{RF})$ & $88.00(\dagger)$ \\
\hline & CAR & $85.53(\mathrm{NB})$ & 99.48 (MLP) & $99.60(\dagger)$ \\
\hline & CHR & $91.75\left(\mathrm{~K}^{*}\right)$ & $99.75(\mathrm{RF})$ & $100.00(\dagger)$ \\
\hline & DER & 86.89 (JRip) & $97.27(\mathrm{NB})$ & $98.09(\dagger)$ \\
\hline & FER & 83.00 (IBk) & 90.00 (MLP) & $92.00(\dagger)$ \\
\hline & FOR & $76.31\left(\mathrm{~K}^{*}\right)$ & 86.46 (SMO) & 87.35 \\
\hline & HEP & 74.84 (JRip) & 85.16 (RF) & 87.71 \\
\hline & LUN & 37.50 (IBk) & $62.50(\mathrm{NB})$ & $69.17(\dagger)$ \\
\hline & SEE & 90.48 (JRip) & 95.24 (MLP) & $97.14(\dagger)$ \\
\hline & SEI & 86.73 (NB) & 93.42 (SMO) & $93.42(\dagger)$ \\
\hline & TEA & 40.40 (JRip) & 66.23 (IBk) & $66.25(\dagger)$ \\
\hline & WIN & $44.24(\mathrm{NB})$ & 70.17 (RF) & $68.58(\dagger)$ \\
\hline & $\mathrm{ABD}$ & 79.46 (IBk) & 86.24 (JRip) & $86.81(\dagger)$ \\
\hline
\end{tabular}

The best combination triad of the 10 aforementioned base classifiers that trained the voting EM has shown higher classification performance ( $2 \%$ on average) compared to the performance of the best individual classifier for most of the databases (25 out of 28) 
(Table 4). Moreover, in 4 datasets (GLA, BER, ECO and LUN) voting achieved an increase in classification performance (based in \% CCI) exceeding 5\%. As for the IRI and SEI databases, the voting performance was found equal to the performance achieved by the best base classifier (MLP and SMO respectively) and finally for the WIN database the voting performance was worse compared with the performance of the RF base classifier. The latter was possibly due to the relatively worse performance of all base classifiers except RF (average performance of the all other classifiers is equal to $56.44 \%$ while the second best classifier lags in performance more than 5\% from RF). The best voting triad for all datasets did not always contain the best base classifier. In fact, in ION, ECO, FOR and HEP datasets the best triad contained, instead of the best base classifier, another base classifier which also achieved high performance (has in average only $1 \%$ lower performance than the best base classifier). However this classifier is much more diverse when compared with the two other members of the classifier triad (JD index increases at least $3 \%$ with this classifier instead of the best base classifier).

\subsection{Results of existing BDIs}

The correlations between the BDIs and the corresponding voting performance (in terms of CCI) of the classifier combinations across training datasets are presented in Table 5. JD can be characterized as the best among BDIs, showing statistically significant correlation $(\mathrm{p}<0.01)$ for most datasets $(11$ out of 14$)$ compared to other indices. Other BDIs such as SMD were more weakly but significantly correlated $(\mathrm{p}<0.01)$ for 10 of the datasets, whereas $\mathrm{Q}$ was significantly correlated $(\mathrm{p}<0.01)$ with CCI only in 8 datasets. The negative correlation between SMD or JD and the voting performance shows that when combining classifiers solely based on their highly dissimilar results (i.e. without taking into account the performance criterion), the subsequent voting performance is reduced. Regarding the sign of the correlation between Q index and CCI, it was found negative in most datasets; in 3 of them (i.e. PAG, VEH and WAV) it was positive with significant correlation, showing that $\mathrm{Q}$ cannot consistently follow the increase of the voting performance. As a result, in some databases the combinations leading to high voting performance correspond to small Q values (negative sign of correlation) while in others to relative large (positive sign). Finally, Phi index was not correlated with voting performance for most of the classifications tasks, therefore it is considered as the least efficient BDI in this study.

Table 5. Spearman rank correlation coefficients of the performance (based on $\mathrm{CCI} \%$ ) of the voting algorithm and the BDIs.

\begin{tabular}{lllll}
\hline Training Database & SMD & JD & Phi & Q \\
\hline ARR & $-0.34^{* *}$ & $-0.44^{* *}$ & $0.19^{*}$ & 0.06 \\
BRE & $-0.39^{* *}$ & $-0.43^{* *}$ & $0.31^{*}$ & $-0.59^{* *}$ \\
GER & -0.17 & $-0.21^{*}$ & 0.12 & $-0.32^{* *}$ \\
GLA & $-0.40^{* *}$ & $-0.46^{* *}$ & $0.31^{* *}$ & -0.09 \\
ION & 0.05 & 0.02 & $-0.29^{* *}$ & $-0.41^{* *}$ \\
IRI & -0.08 & 0.04 & -0.11 & $-0.45^{* *}$
\end{tabular}




\begin{tabular}{lllll} 
LAB & $-0.34 * *$ & $-0.40^{* *}$ & -0.11 & -0.07 \\
PAG & $-0.70^{* *}$ & $-0.71 * *$ & $0.66 * *$ & $0.18^{*}$ \\
SEG & $-0.72 * *$ & $-0.72 * *$ & 0.34 & -0.18 \\
VEH & $-0.24 * *$ & $-0.38^{* *}$ & 0.01 & $0.34 * *$ \\
VOT & $-0.60^{* *}$ & $-0.60 * *$ & $0.35 * *$ & $-0.37 * *$ \\
WAV & $-0.61 * *$ & $-0.62 * *$ & $0.56 * *$ & $0.52 * *$ \\
BER & $-0.53 * *$ & $-0.62 * *$ & 0.00 & $-0.39 * *$ \\
ECO & $-0.21 *$ & $-0.41 * *$ & 0.16 & -0.15 \\
\hline
\end{tabular}

* Significant correlation at the 0.05 level (2-tailed)

** Significant correlation at the 0.01 level (2-tailed)

\subsection{Optimizing the DP index}

DP index incorporates two basic criteria (i.e. individual performance and the dissimilarity of base classifiers) by weighting them with parameter $\alpha$ (section 2.5). Since JD was the best performing BDI (showing the best correlation with voting performance across databases), it was selected to represent the dissimilarity among classifier results in DP index. In order to select the optimal value of parameter $a$, DP was computed along an $\alpha$ range from 0 to 1 with 0.01 step for all datasets. Subsequently, the correlation coefficient between DP and voting performance was computed along the parameter $\alpha$ range for all datasets (Fig. 2). The graph shows that for small parameter $\alpha$ values (i.e. the criterion of dissimilarity among classifiers results prevails in DP), the correlation coefficient starts with high negative values and approaches zero as the value of $a$ increases (the criterion of individual performance of base classifiers getting into DP). Thereafter, the correlation coefficient changes sign and increases gradually reaching a maximum value when $a$ is within a relative small interval (between 0.7 and 0.8 for all datasets). Above this point the correlation decreases as $a$ approaches 1 (i.e. only individual classifier performance remains in DP). The aforementioned behavior was observed across all training datasets, except of IRI in which the correlation increases constantly when $a$ parameter increases. This inconsistency can be explained by the fact that in this database the base classifiers had very similar performances (CCI equal to $94 \%$ and $97.33 \%$ for the worst and best classifier) (Table 4) and also by the negligible dissimilarity among the classifier results (mean JD of the 10 classifiers equal to 0.03 ). 


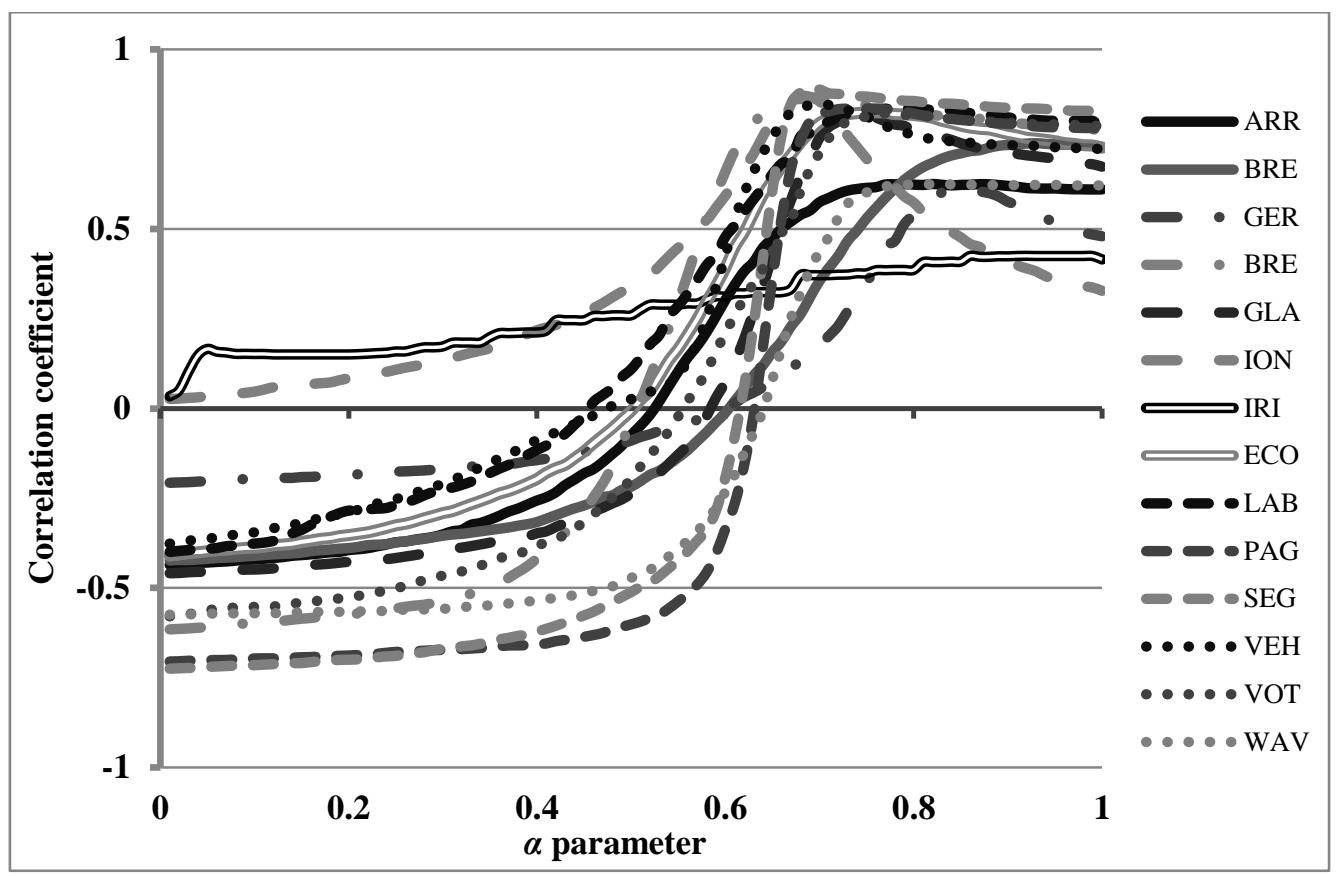

Fig. 2. Change of correlation coefficient between voting performance and DP index for different values of parameter $\alpha$ for each training dataset.

The optimal values of parameter $a$ (maximizing the correlation between DP and voting performance for each dataset) had relatively small variation $(n=14$, variance $=0.004$, coefficient of variation=8\%) showing that the optimal value of parameter $a$ does not considerably vary among datasets. Thus, the mean of optimal values of $a$ estimated from all datasets was used as the most appropriate measure to determine the $a$ value within DP index $(a=0.77)$. The DP index was thus computed by fixing the value of parameter $a$ at 0.77 .

\subsection{Efficiency of DP index}

The correlation between DP and voting performance (in terms of CCI) for the 120 different classifier triads for all datasets is presented in Table 6. DP correlated $(\mathrm{p}<0.01)$ with voting performance for both training and testing datasets and the correlation coefficients were considerably high $(\mathrm{R}>0.70)$ in most databases. In three datasets (i.e. IRI, GER and HEP) the correlation was rather weak but still statistically significant $(\mathrm{p}<0.01)$. Considering that voting performance can be expressed by $\% \mathrm{CCI}$, the most efficient among the considered indices is DP showing consistently positive and higher correlations for all datasets. Moreover, the 12 triads (10\% of the total) with the highest DP values for each testing dataset were those having the corresponding higher 
performance during voting EM. The optimal triad (i.e. the one with the best voting performance) was found amongst the selected triads across testing (also for training) datasets (tick symbol in Table 6) and thus DP can be considered effective in identifying the best classifier combination.

\begin{tabular}{|c|c|c|c|}
\hline Training Database & $\mathrm{DP}(\alpha=0.77)$ & Testing Database & $\mathrm{DP}(\alpha=0.77)$ \\
\hline ARR & $0.63 * *(\dagger)$ & BAN & $0.81 * *(\dagger)$ \\
\hline BRE & $0.59 * *(\dagger)$ & BLO & $0.88 * *(\dagger)$ \\
\hline GER & $0.45 * *(\dagger)$ & CAR & $0.82 * *(\dagger)$ \\
\hline GLA & $0.80 * *(\dagger)$ & CHR & $0.89 * *(\dagger)$ \\
\hline ION & $0.64 * *(\dagger)$ & DER & $0.74 * *(\dagger)$ \\
\hline IRI & $0.39 * *(\dagger)$ & FER & $0.66 * *(\dagger)$ \\
\hline LAB & $0.84 * *(\dagger)$ & FOR & $0.55 * *(\dagger)$ \\
\hline PAG & $0.83 * *(\dagger)$ & HEP & $0.46 * *(\dagger)$ \\
\hline SEG & $0.86^{* *}(\dagger)$ & LUN & $0.64 * *(\dagger)$ \\
\hline VEH & $0.79 * *(\dagger)$ & SEE & $0.81 * *(\dagger)$ \\
\hline VOT & $0.82 * *(\dagger)$ & SEI & $0.65 * *(\dagger)$ \\
\hline WAV & $0.66^{* *}(\dagger)$ & TEA & $0.80 * *(\dagger)$ \\
\hline BER & $0.84 * *(\dagger)$ & WIN & $0.70 * *(\dagger)$ \\
\hline $\mathrm{ECO}$ & $0.83 * *(\dagger)$ & $\mathrm{ABD}$ & $0.75 * *(\dagger)$ \\
\hline
\end{tabular}

The monotonic behavior of DP was checked by plotting its variability (120 classifier triads implemented in 14 datasets $=120 * 14=1680$ points) in relation to the voting performance in terms of CCI (Fig. 3) for comparative reasons. DP has shown consistent increase along the voting CCI spectrum and thus its behavior is monotonic. The few points in which the estimated DP index was rather high (4-5 points at the left side) represent classifier triads in which one poorly performing classifier participates. In those few cases, the voting performance was greatly affected by the contribution of a poor classifier (i.e. NB for WIN dataset or IBk for LUN dataset) but DP could not incorporate such effects. However, the estimated correlation coefficient between DP and voting performance was extremely high for all points $(n=1680$, Spearman $R=0.99, p<0.01)$ indicating the monotonic increase of DP across the corresponding voting performance increase expressed by \% CCI. More specifically, the triads having high DP values (being of high importance because they can demonstrate the optimal classifier combinations) are those that finally achieved the best voting performances (points located on the top right of the graph). 


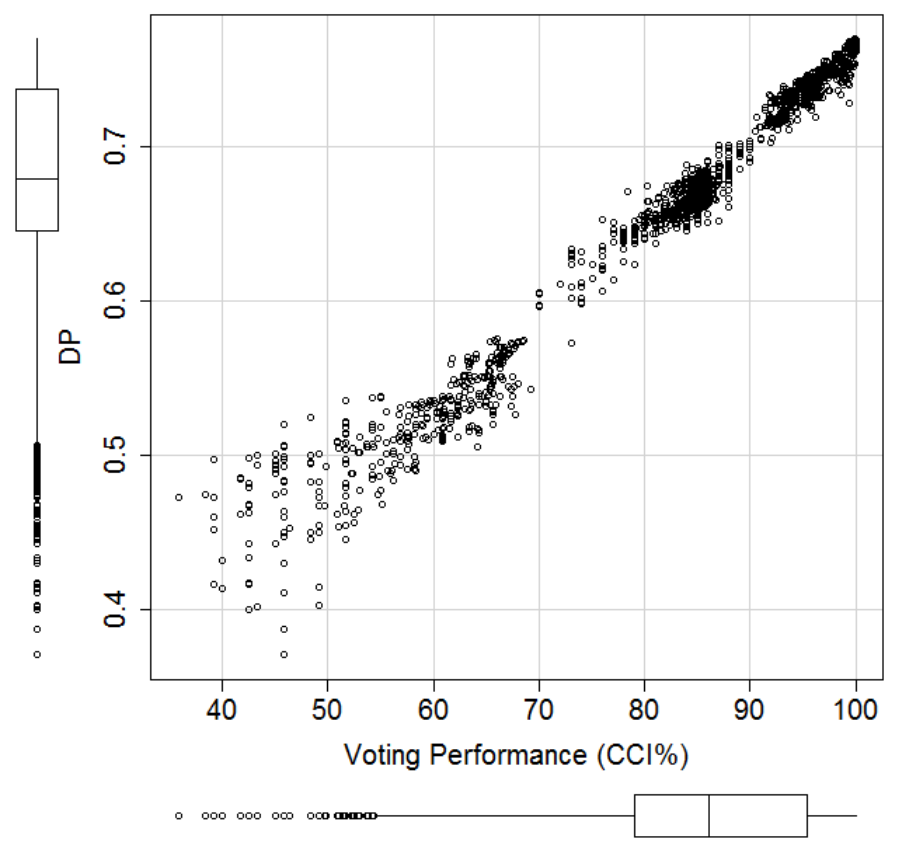

Fig. 3. Monotonic behavior of DP along voting performance in terms of CCI\% gradient for all classifier triads across training datasets ( 120 classifier combinations $* 14$ datasets $=1680$ points)

The percentage of improvement in voting performance when trained with the DP proposed triad against the best base classifier across different number of labels of the target class is presented in Fig 4. This improvement in performance is approximately 1$2 \%$ for databases with 2, 3, 4 and more than 6 labels and relatively higher (around 4\%) for databases with 5 or 6 labels. Thus, DP offers significant performance improvement regardless of the number of labels of the target class of the trained dataset.

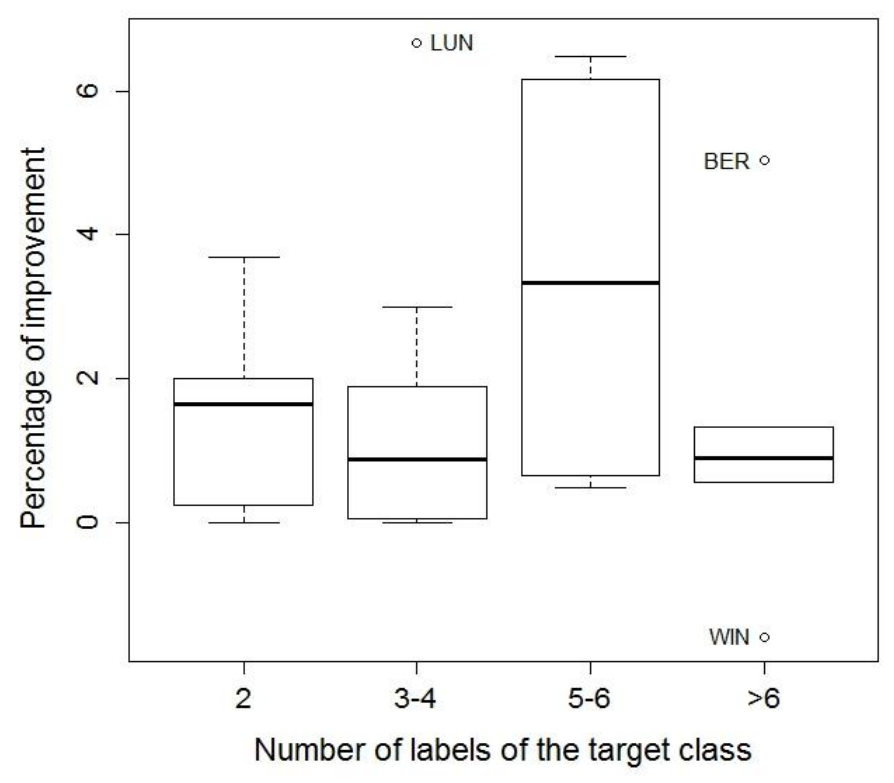


Fig. 4. Percentage of improvement achieved by voting EM compared to the best base classifier in accordance with the number of labels of the target class of each dataset.

\section{Discussion}

In the present study, 10 base classifiers corresponding to various ML categories, were trained using 28 substantially different datasets from diverse scientific fields (e.g. Medicine, Ecology, Botany, Physics, Sociology, Economy, Web, and Engineering) in order to access their individual classifying efficiency. MLP was the best performing base classifier in contrast to NB which showed the poorest performance in agreement with previous applications. ${ }^{98-100}$ Subsequently, all possible classifier triads resulting from combinations of the 28 base classifiers were used to train the easiest and most widely applicable EM i.e. voting classifier. The best triad has shown improved performance across most datasets (except for IRI, SEI and WIN databases in which the voting performance was either equal or slightly worse compared with the best base classifier) in agreement with the general principle that ensembles of classifiers are often substantially more accurate than their individual base classifiers. ${ }^{20,27,29,40,101}$ For 4 datasets the $\%$ increase of CCI was over 5\%, considered as a remarkable improvement in classification performance. $^{12}$

Each base classifier employs a different learning strategy to provide its own classification results which are fed into the voting algorithm for the final classification. When the individual results are similar then the outcome will be based more or less on the same information (errors and corrects), without offering any additive value in voting, increasing however the system complexity. ${ }^{25,26,35,102}$ On the other hand, EMs consisting of classifiers offering different results have the potential to achieve significantly better performance compared to those of individual base classifiers. ${ }^{15,19,35,39,103}$ This finding was confirmed in the present study, with SMC and JD dissimilarity measures showing statistically significant correlations with voting performance for most of the training datasets. More specifically JD scored higher (absolute) values of correlation than the comparable SMC index rather because it emphasizes on differences between classifier results. ${ }^{104}$ However, the two measures of independency (i.e. Q and Phi) that were supposed to improve voting accuracy, ${ }^{17,76,105}$ showed low correlation with voting performance also in agreement with some previous studies. ${ }^{35,36,38}$

Although dissimilarity among individual classifiers combined to develop EMs may be the key towards improving classification efficiency ${ }^{88}$ dissimilar but powerless classifiers are unlikely to bring any benefits in EMs performance. ${ }^{35,106}$ The latter was confirmed in the present study by the negative sign of correlation indicating that classifier triads with highly differentiated results (as expressed by JD or SMC) often contain at least one poor classifier which significantly affects the voting performance. In addition, the relative high correlation between the voting performance and the individual performances (when $a=1$ in DP) of base classifiers denoted for the training datasets, indicates that the individual performance is a rather crucial characteristic in optimizing EMs as previously 
suggested. ${ }^{18,107,108}$ Thus, both accurate and diverse classifiers are needed in order to construct combinations that optimize the voting performance.

These two crucial characteristics, dissimilarity and individual performance of combined classifiers, have been integrated in the present study in a new index selecting the optimum classifier combinations to train the voting algorithm. The DP index integrates dissimilarity using the JD measure, which is generally considered as an efficient and stable indicator. ${ }^{109}$ In the present study JD was the most sensitive among the BDIs on following the voting performance variability. This is in agreement with Kuncheva and Hadjitodorov ${ }^{110}$ who employed JD in cluster ensembles. In addition, DP index integrates the performance characteristic using the individual performance of the classifiers, as it is widely accepted and also proved in the present study that optimal combinations should include classifiers with high individual performances. ${ }^{24,47}$

The dissimilarity and the individual performance of classifier combinations were weighted in DP using parameter $\alpha$. The optimal value of this parameter (maximizing the correlation between DP and voting performance) did not vary significantly among the 14 substantially different training datasets suggesting that the DP performance is consistent and robust across different classification tasks. Subsequently, the determined DP outperformed all BDIs by showing statistically significant correlation with the voting performance across all training datasets. The same correlation was also found statistically significant for all testing datasets, the values of the correlation coefficient being relatively high ( $\mathrm{R}>0.7$ in most cases). These findings show that DP index is both efficient and consistent, being highly correlated with voting performance. Moreover, the optimization of DP offered a prioritization between the two characteristics of the classifier combinations with the dissimilarity weighting almost three times less than the individual performance $(a=0.77)$. This prioritization makes DP efficient in determining the best performing classifier triad during voting for all datasets, even for those (ION, ECO, FOR and HEP) where the best triad did not contain the base classifier with the best individual performance. In these datasets, the best triads contain classifiers with relatively lower performance compared to the best, however with sufficiently dissimilar results which maximize DP value. This finding shows that DP performs better than methods proposing triads including by default the best performing base classifier. ${ }^{41}$ Furthermore, DP showed a monotonic increase across the voting performance spectrum $(R>0.95)$ regardless of the testing dataset suggesting that it closely matches the voting performance. This matching was achieved even for datasets where voting EM performed lower than the best base classifier (i.e. WIN), in which although DP managed to determine the best triad. Finally, DP offered significant improvement (as least 1\%) in the ensemble performance regardless of the number of labels of the target class.

\section{Conclusions}


In this paper, a new index named DP was proposed in order to rank the classifier combinations according to their performance during voting training. Based on the simulation results, comparisons and discussion we have the following conclusions:

(1) Both individual performance and dissimilarity in classification outcomes when classifiers participate in voting are crucial criteria affecting the voting performance.

(2) DP which optimally incorporates the above characteristics (i.e. individual performance and dissimilarity) achieved an efficient ranking of classifier combinations according to their voting performance.

DP is a useful tool for the individual users aiming to identify the optimal classifier combinations to use in voting EM, in order to easily achieve improved classification performance using their own familiar and tested ML algorithms. Apart from the easiness of application, DP has a number of additional advantages i.e. simplicity (it uses only three combined classifiers), efficiency (it successfully selects the classifiers to participate in the voting algorithm), flexibility (any base classifier can be included in the DP computation) and consistency (robust performance across substantially different datasets). On the other hand, DP cannot be compared with EM schemes that perform thorough search towards inducing all possible kinds of classification errors which however need qualified designers to apply them to new tasks. Finally, in datasets where the training of voting EM results in lower performance compared to the individual base classifiers, DP still defines the optimal classifier triad which however is not improving the performance of the requested classification issue. 


\section{References}

1. J. G. Carbonell, R. S. Michalski and T. M. Mitchell, An overview of machine learning, in Machine Learning, eds. R. S. Michalski, J. G. Carbonell and T. M. Mitchell (Springer, Berlin/Heidelberg, 1983), pp. 3-23.

2. S. B. Kotsiantis, I. D. Zaharakis and P. E. Pintelas, Machine learning: a review of classification and combining techniques, Artificial Intelligence Review 26(3) (2006) 159-190.

3. C. Apte, R. Sasisekharan, V. Seshadri and S. M. Weiss, Case studies in high-dimensional classification, Applied Intelligence 4(3) (1994) 269-281.

4. G. Huang, G. B. Huang, S. Song and K. You, Trends in extreme learning machines: a review, Neural Networks 61 (2015) 32-48.

5. M. Kuramochi and G. Karypis, Gene classification using expression profiles: a feasibility study, International Journal of Artificial Intelligence Tools 14(4) (2005) 641-660.

6. Y. Shi, Y. Gao, R. Wang, Y. Zhang and D. Wang, Transductive cost-sensitive lung cancer image classification, Applied Intelligence 38(1) (2013) 16-28.

7. I. Koprinska, J. Poon, J. Clark and J. Chan, Learning to classify e-mail, Information Sciences 177(10) (2007) 2167-2187.

8. M. Brescia, S. Cavuoti, M. Paolillo, G. Longo and T. Puzia, The detection of globular clusters in galaxies as a data mining problem, Monthly Notices of the Royal Astronomical Society 421(2) (2012) 1155-1165.

9. G. Tsekouras, H. Sarimveis, C. Raptis and G. Bafas, A fuzzy logic approach for the classification of product qualitative characteristics, Computers \& Chemical Engineering 26(3) (2002) 429-438.

10. K. Topouzelis and D. Kitsiou, Detection and classification of mesoscale atmospheric phenomena above sea in SAR imagery, Remote sensing of environment 160 (2015) 263-272.

11. D. Lu and Q. Weng, A survey of image classification methods and techniques for improving classification performance, International Journal of Remote Sensing 28(5) (2007) 823-870.

12. M. Pal and P. M. Mather, An assessment of the effectiveness of decision tree methods for land cover classification, Remote Sensing of Environment 86 (4) (2003) 554-565.

13. N. Japkowicz, Supervised versus unsupervised binary-learning by feedforward neural networks, Machine Learning 42(1-2) (2001) 97-122.

14. S. B. Kotsiantis, Supervised learning: A review of classification techniques, Informatica 31 (2007) 249-268.

15. L. I. Kuncheva, Combining pattern classifiers: methods and algorithms, 2nd edn. (Wiley, New Jersey, 2014).

16. B. B. Chaudhuri and U. Bhattacharya, Efficient training and improved performance of multilayer perceptron in pattern classification, Neurocomputing 34(1-4) (2000) 11-27.

17. P. Baldi, S. Brunak, Y. Chauvin, C. A. F. Andersen and H. Nielsen, Assessing the accuracy of prediction algorithms for classification: An overview, Bioinformatics 16(5) (2000) 412 424.

18. D. W. Opitz and R. Maclin, Popular ensemble methods: an empirical study, Journal of Artificial Intelligence Research 11 (1999) 169-198. 
19. Z. Liu, Q. Dai and N. Liu, Ensemble selection by GRASP, Applied Intelligence 41(1) (2014) 128-144.

20. Z. H. Zhou, Ensemble methods - Foundations and algorithms, (Chapman \& Hall/CRC, Florida, 2012).

21. C. N. Anagnostopoulos, T. Iliou and I. Giannoukos, Features and classifiers for emotion recognition from speech: a survey from 2000 to 2011, Artificial Intelligence Review 42(2) (2012) 1-23.

22. M. Wozniak, M. Grana and E. Conchado, A survey of multiple classifier systems as hybrid systems, Information Fusion 16 (2014) 3-17.

23. E. Bauer and R. Kohavi, An empirical comparison of voting classification algorithms: bagging, boosting, and variants. Machine Learning 36(1-2) (1999) 105-139.

24. T. G. Dietterich, An experimental comparison of three methods for constructing ensembles of decision trees: bagging, boosting, and randomization, Machine Learning 40(2) (2000) 139157.

25. A. C. Tan and D. Gilbert, Ensemble machine learning on gene expression data for cancer classification, Applied Bioinformatics 2 (2003) S75-S83.

26. A. Ulas, M. Semerci, O. T. Yildiz and E. Alpaydin, Incremental construction of classifier and discriminant ensembles, Information Sciences 179(9) (2009) 1298-1318.

27. A. Onan, S. Korukoglu and H. Bulut, A multiobjective weighted voting ensemble classifier based on differential evolution algorithm for text sentiment classification, Expert Systems with Applications 62 (2016) 1-16.

28. G. Tsoumakas, I. Katakis and I. Vlahavas, Effective Voting of Heterogeneous Classifiers, in: Machine Learning: ECML 2004, eds. J. F. Boulicaut, F. Esposito, F. Giannotti, D. Pedreschi (Springer, Berlin/Heidelberg, 2004) pp. 465-476.

29. S. Saha and A. Ekbal, Combining multiple classifiers using vote based classifier ensemble technique for named entity recognition, Data \& Knowledge Engineering 85 (2013) 15-39.

30. N. Dimililer, E. Varoglu and H. Altincay, Classifier subset selection for biomedical named entity recognition, Applied Intelligence 31(2009) 267-282.

31. E. Dimitriadou, A. Weingessel and K. Hornik, Voting-Merging: An ensemble method for clustering, in: Artificial Neural Networks-ICANN 2001, eds. G. Dorffner, H. Bischof, K. Hornik , (Springer, Berlin/Heidelberg, 2001), pp. 217-224.

32. E. Loza Mencia, S. H. Park and J. Furnkranz, Efficient voting prediction for pairwise multilabel classification, Neurocomputing 73(7-9) (2010) 1164-1176.

33. H. Ishibuchi, T. Nakashima and T. Morisawa, Voting in fuzzy rule-based systems for pattern classification problems, Fuzzy Sets and Systems 103(2) (1999) 223-238.

34. V. G. Kaburlasos and T. Pachidis, A Lattice-Computing ensemble for reasoning based on formal fusion of disparate data types, and an industrial dispensing application, Information Fusion 16 (2014) 68-83.

35. D. Ruta and B. Gabrys, Classifier selection for majority voting, Information Fusion 6(1) (2005) 63-81.

36. R. E. Banfield, L. O. Hall, K. W. Bowyer and W. P. Kegelmeyer, Ensemble diversity measures and their application to thinning, Information Fusion 6(1) (2005) 49-62. 
37. L. I. Kuncheva and C. J. Whitaker, Measures of diversity in classifier ensembles and their relationship with the ensemble accuracy, Machine Learning 51(2) (2003) 181-207.

38. A. Shipp and L. I. Kuncheva, Relationships between combination methods and measures of diversity in combining classifiers, Information Fusion 3(2) (2002) 135-148.

39. I. Perikos and I. Hatzilygeroudis, Recognizing emotions in text using ensemble of classifiers, Engineering Applications of Artificial Intelligence 51 (2016) 191-201.

40. G. Rudolph and T. Martinez, Finding the real differences between learning algorithms, International Journal on Artificial Intelligence Tools 24(3) (2015).

41. A. Tamvakis, G. E. Tsekouras, A. Rigos, C. Kalloniatis, C. N. Anagnostopoulos and G. Anastassopoulos, A methodology to carry out voting classification tasks using a particle swarm optimization-based neuro-fuzzy competitive learning network, Evolving Systems 8(1) (2017) 49-69.

42. M. Amini, J. Rezaeenour and E. Hadavandi, A neural network ensemble classifier for effective intrusion detection using fuzzy clustering and radial basis function networks, International Journal on Artificial Intelligence Tools 25(2) (2016).

43. Z. H. Zhou, Clusterer ensemble, Knowledge-Based Systems 19(1) (2006) 77-83.

44. J. S. Chou, C. F. Tsai, A. D. Pham and Y. H. Lu, Machine learning in concrete strength simulations: Multi-nation data analytics, Construction and Building Materials 73 (2014) 771780.

45. F. Roli and G. Giacinto, Design of multiple classifier systems, in: Hybrid Methods in Pattern Recognition, eds. H. Bunke and A. Kandel, (Worldwide Scientific Publishing, Singapore, 2002) pp 199-226.

46. N. Garcia-Pedrajas, J. Maudes-Raedo, C. Garcia-Osorio and J. J. Rodriguez-Diez, Supervised subspace projections for constructing ensembles of classifiers, Information Sciences 193 (2012) $1-21$.

47. G. Giacinto and F. Roli, An approach to the automatic design of multiple classifier systems, Pattern Recognition Letters 22(1) (2001) 25-33.

48. W. Opitz and J. W. Shavlik, Actively searching for an effective neural network ensemble, Connection Science 8(3-4) (1996) 337-353.

49. M. Hall, E. Frank, G. Holmes, B. Pfahringer, P. Reutemann and I. H. Witten, The WEKA data mining software: an Update, SIGKDD Explorations 11(1) (2009) 10-18.

50. F. Burkhardt, A. Paeschke, M. Rolfes, W. Sendlmeier and B. Weiss, A database of German emotional speech. in: Proc. 9th Int. Conf. on Spoken Language Processing (INTERSPEECHICSLP, Lissabon, 2005) pp. 1517-1520.

51. P. Boersma and D. Weenink, Praat: doing phonetics by computer (version 4.6.09) (2005), http://www.praat.org.

52. N. Anagnostopoulos and T. Iliou, Towards emotion recognition from speech: definition, problems and the materials of research, in: Semantics in Adaptive and Personalized Services, eds. M. Wallace, I. Anagnostopoulos, P. Mylonas and M. Bielikova, (Springer, Berlin/Heidelberg, 2010), pp. 127-143. 
53. S. Spatharis, G. Tsirtsis, D. B. Danielidis, D. C. Thang and D. Mouillot, Effects of pulsed nutrient inputs on phytoplankton assemblage structure and blooms in an enclosed coastal area, Estuarine, Coastal and Shelf Science 73(3-4) (2007) 807-815.

54. N. Simboura, P. Panayotidis and E. Papathanassiou, A synthesis of the biological quality elements for the implementation of the European Water Framework Directive in the Mediterranean ecoregion: The case of Saronikos Gulf, Ecological Indicators 5(3) (2005) 253266.

55. A. Tamvakis, C. N. Anagnostopoulos, G. Tsekouras and G. Anastassopoulos, Optimizing voting classification using cluster analysis on medical diagnosis data, in: Proc. 16th Int. Conf. on Engineering Applications of Neural Networks (EANN'15, Rhodes, Greece, 2015) pp. 1-7.

56. A. Deshmukh, A. S. Patil and B. V. Pawar, Comparison of classification algorithms using WEKA on various datasets, International Journal of Computer Science and Information Technology 4(2) (2011) 85-90.

57. R. C. Holte, Very simple classification rules perform well on most commonly used datasets, Machine Learning 11(1) (1993) 63-90.

58. M. Varna and R. B. Bolda, More generality in efficient multiple Kernal learning, in: Proc. 26th Annual Int. Conf. on Machine Learning (ICML, Montreal, Quebec, Canada) (2009) pp. 1062-1072.

59. S. W. Lin and S. C. Chen, PSOLDA: A particle swarm optimization approach for enhancing classification accuracy rate of linear discriminant analysis, Applied Soft Computing 9 (2009) 1008-1015.

60. S. Ozsoy, G. Gumus and S. Khalilov, C4.5 versus other decision trees: A review, Computer Engineering and Applications 4 (2015) 173-181.

61. I. Sangaiah, A. V. A. Kumar, A. Balamurugan, An empirical study on different ranking methods for effective data classification, Journal of Modern Applied Statistical Methods 14(2) (2015) 35-52.

62. A. Iranzad, A. Masoudnia, F. Cheraghchi, A. Nowzari-Dalini, R. Ebrahimpour, Improving combination methods of neural classifiers using NCL, International Journal of Computer Information Systems and Industrial Management Applications 4 (2012) 679-686

63. D. Koller and M. Sahami, Toward optimal feature selection, in: Proc. 13th Int. Conf. on Machine Learning (Bari, Italy, 1996) pp. 284-292.

64. J. Wang and G. Karypis, HARMONY: Efficiently mining the best rules for classification, in: Proc. 5th Int. Conf. on Data Mining (SIAM, California, 2005) pp. 205-216.

65. N. I. Nife, Performance analaysis of various data mining techniques on banknote authentication, International Journal of Engineering Science Invention 5(2) (2016) 62-71.

66. Y. Asim, A. R. Shahid, A. K. Malik, B. Raza, Significance of machine learning algorithms in professional blogger's classification, Computers and Electrical Engineering (In Press 2017) $1-33$.

67. A. Selekin and J. Stankovic, Detection of chronic kidney disease and selecting important predictive attributes, in: IEEE Int. Conf. on Healthcare Informatics (ICHI, Chicago, 2016) pp. 1-9. 
68. D. Gil, J. L. Girela, J. De Juan, M. J. Gomez-Torres, and M. Johnsson, Predicting seminal quality with artificial intelligence methods, Expert Systems with Applications 39(16) (2012) 12564-12573.

69. B. Johnson, R. Tateishi and Z. Xie, Using geographically-weighted variables for image classification, Remote Sensing Letters 3(6) (2012) 491-499.

70. V. Singh, L. Mukherjee, J. Peng and J. Xu, Ensemble clustering using semidefinite programming with applications, Machine Learning 79 (2010) 177-200.

71. M. Charytanowicz, J. Niewczas, P. Kulczycki, P. A. Kowalski, S. Lukasik and S. Zak, Complete gradient clustering algorithm for features analysis of x-ray images, in: Information Technologies in Biomedicine, eds. E. Pietka, J. Kawa (Springer, Berlin/Heidelberg, 2010) pp. 15-24.

72. D. Bertsimas and J. Dunn, Optimal classification trees, Machine Learning 106 (2017) 10391082.

73. P. Cortez, A. Cerdeira, F. Almeida, T. Matos and J. Reis, Modeling wine preferences by data mining from physicochemical properties, Decision Support Systems 47 (2009) 547-533.

74. T. Iliou, C. N. Anagnostopoulos, I. Stephanakis and G. Anastassopoulos, Combined classification of risk factors for appendicitis prediction in childhood, in: Engineering Applications of Neural Networks, eds. L. Iliadis, H. Papadopoulos and C. Jayne, (Springer, Berlin/Heidelberg, 2013), pp. 203-211.

75. S. Dzeroski and B. Zenko, Is combining classifiers with stacking better than selecting the best one?, Machine Learning 54(3) (2004) 255-273.

76. L. I. Kuncheva, C. J. Whitaker, C. A. Shipp and R. P. W. Duin, Is independence good for combining classifiers?, in: Proc.15th Int. Conf. on Pattern Recognition, (Barcelona, 2000), pp. 168-171.

77. G. Zhou, D. Shen, J. Zhang, J. Su and S. Tan, Recognition of protein/gene names from text using an ensemble of classifiers, BMC Bioinformatics 6 (2005) S7.

78. W. W. Cohen, Fast effective rule induction, in: Proc. 12th Int. Conf. on Machine Learning, (Morgan Kaufmann, San Francisco, 1995), pp.115-123.

79. H. Witten and E. Frank, Generating accurate rule sets without global optimization, in: Proc. 15th Int. Conf. on Machine Learning, (Morgan Kaufmann, San Francisco, 1998) pp. 144-151.

80. J. R. Quinlan, C4.5: Programs for Machine Learning, (Morgan Kaufmann, San Francisco, 1993).

81. L. Breiman, Random Forests, Machine Learning 45(1) (2001) 5-32.

82. W. Aha, D. Kibler and M. K. Albert, Instance-based learning algorithms, Machine Learning 6(1) (1991) 37-66.

83. J. G. Cleary and L. E. Trigg, K*: An instance-based learner using an entropic distance measure, in: Proc.12th Int. Conf. on Machine Learning, (Morgan Kaufmann, San Francisco, 1995), pp. 108-114.

84. S. le Cassie and J. C. van Houwelingen, Ridge estimators in logistic regression, Applied Statistics 41 (1992) 191-201.

85. S. K. Pal and S. Mitra, Multilayer perceptron, fuzzy sets, and classification, IEEE Transactions on Neural Networks 3 (1992) 683-697. 
86. J. C. Platt, Fast training of support vector machines using sequential minimal optimization, in: Advances in Kernel Methods-Support Vector Learning, eds. B. Scholkopf, C. J. C. Burges and A. Smola, (MIT Press, Cambridge USA, 1998), pp. 185-208.

87. H. John and P. Langley, Estimating continuous distributions in Bayesian classifiers, in: Proc. 11th Conf. on Uncertainty in Artificial Intelligence, (Morgan Kaufmann, San Francisco, 1995), pp. 338-345.

88. J. Kittler, M. Hatef, R. W. D. Duin, and J. Matas, On combining classifiers, IEEE Transactions on Pattern Analysis and Machine Intelligence 20 (3) (1998) 226-239.

89. M. Stone, Cross-validation and multinomial prediction, Biometrika 6(3) (1974) 509-515.

90. I. H. Witten and E. Frank, Data Mining - Practical Machine Learning Tools and Techniques, (Morgan Kaufmann, San Francisco, 2005).

91. T. H. Hoang, K. Lock, A. Mouton and P. L. M. Goethals, Application of classification trees and support vector machines to model the presence of macroinvertebrates in rivers in Vietnam, Ecological Informatics 5(2) (2010) 140-146.

92. R. R. Sokal and P. H. A. Sneath, Principles of numerical taxonomy, (W. H. Freeman, San Francisco, 1963).

93. P. Jaccard, Nouvelles recherctus sur la distribution floral, Bulletin Society Science Naturale 44 (1908) 223-270.

94. U. Yule, On the methods of measuring the association between two variables, Journal of the Royal Statistical Society 75 (1912) 579-642.

95. G. U. Yule, On the association of attributes in statistics. Philosophy of Transactions A 194 (1900) 257-319.

96. A. J. C. Sharkey and N. E. Sharkey, Combining diverse neural nets, The Knowledge Engineering Review 12(3) (1997) 231-247.

97. S. Spatharis and G. Tsirtsis, Ecological quality scales based on phytoplankton for the implementation of Water Framework Directive in the Eastern Mediterranean, Ecological Indicators 10(4) (2010) 840-847.

98. J. Boccard, A. Kalousis, M. Hilario, P. Lanteri, M. Hanafi, G. Mazerolles, J. L. Wolfender, P. A. Carrupt and S. Rudaz, Standard machine learning algorithms applied to UPLC-TOF/MS metabolic fingerprinting for the discovery of wound biomarkers in Arabidopsis thaliana, Chemometrics and Intelligent Laboratory Systems 104(1) (2010) 20-27.

99. I. De Falco, A. Della Cioppa and E. Tarantino, Automatic classification of handsegmented image parts with differential evolution, in: Applications of Evolutionary Computing, eds. F. Rothlauf, J. Branke, S. Cagnoni, E. Costa, C. Cotta, R. Drechsler, E. Lutton, P. Machado, J. Moore, J. Romero, G. Smith, G. Squillero and H. Takagi, (Springer, Berlin Heidelberg, 2006) pp. 403-414.

100. M. Roshandel, A. Munjal, P. Moghadam, S. Tajik and H. Ketabdar, Multi-sensor finger ring for authentication based on 3D signatures, in: 16th Int. Conf. Human-Computer Interaction, Advanced Interaction Modalities and Techniques, ed. M. Kurosu, (Springer, 2014), pp. 131138.

101. T. G. Dietterich, Machine learning research: Four current directions, AI Magazine 18(4) (1997) 97-136. 
102. A. Tsymbal, M. Pechenizkiy and P. Cunningham, Diversity in search strategies for ensemble feature selection, Information Fusion 6(1) (2005) 83-98.

103. M. P. Canuto, M. C. C. Abreu, L. de Melo Oliveira, J. C. Jr. Xavier and A. de M. Santos, Investigating the influence of the choice of the ensemble members in accuracy and diversity of selection-based and fusion-based methods for ensembles, Pattern Recognition Letters 28 (4) (2007) 472-486.

104. H. Cheetham and J. E. Hazel, Binary (presence-absence) similarity coefficients, Journal of Paleontology 43 (1969) 113-136.

105. I. Kuncheva, C. J. Whitaker, C. A. Shipp and R. P. W. Duin, Limits on the majority vote accuracy in classifier fusion, Pattern Analysis \& Applications 6(1) (2003) 22-31.

106. S. Mao, L. C. Jiao, L. Xiong and S. Gou, Greedy optimization classifiers ensemble based on diversity, Pattern Recognition 44 (6) (2011) 1245-1261.

107. G. Rogova, Combining the results of several neural network classifiers, Neural Networks 7(5) (1994) 777-781.

108. T. Rohlfing, D. B. Russakoff and C. R. Maurer, Performance-based classifier combination in atlas-based image segmentation using expectation-maximization parameter estimation, IEEE Transactions on Medical Imaging 23(8) (2004) 983-994.

109. Y. Yin and K. Yasuda, Similarity coefficient methods applied to the cell formation problem: a comparative investigation, Computers \& Industrial Engineering 48(3) (2005) 471-489.

110. I. Kuncheva, S. T. Hadjitodorov, Using diversity in cluster ensembles, in: IEEE Int. Conf. on Systems, Man and Cybernetics, (Hague, 2004) pp. 1214-1219. 


\section{Figure captions}

Fig. 1. Schematic diagram of the methodological steps followed in the development and testing of the proposed Dissimilarity-Performance (DP) index. This index takes into account both the individual performance of base classifiers (P) and the dissimilarity (D) of classifier performance -measured with Binary Dissimilarity Indices (BDIs) - when those are combined in triads.

Fig. 2. Change of correlation coefficient between voting performance and DP index for different values of parameter $\alpha$ for each training dataset.

Fig. 3. Monotonic behavior of DP along voting performance in terms of CCI \% gradient for all classifier triads across training datasets (120 classifier combinations * 14 datasets $=1680$ points) 